\title{
Several properties of hypergeometric Bernoulli numbers
}

\author{
Miho Aoki ${ }^{1}$, Takao Komatsu ${ }^{2 *}$ (D) and Gopal Krishna Panda ${ }^{3}$
}

\section{"Correspondence:}

komatsu@zstu.edu.cn

${ }^{2}$ Department of Mathematical

Sciences, School of Science,

Zhejiang Sci-Tech University,

Hangzhou, China

Full list of author information is

available at the end of the article

\begin{abstract}
In this paper, we give several characteristics of hypergeometric Bernoulli numbers, including several identities for hypergeometric Bernoulli numbers which the convergents of the continued fraction expansion of the generating function of the hypergeometric Bernoulli numbers entail. We show an analog of Kummer's congruences in the classical Bernoulli numbers. We also give some determinant expressions of hypergeometric Bernoulli numbers and some relations between the hypergeometric and the classical Bernoulli numbers. By applying Trudi's formula, we have some different expressions and inversion relations.
\end{abstract}

MSC: 11A55; 11B68; 11B37; 11C20; 15A15; 33C15; 05A15; $05 \mathrm{~A} 19$

Keywords: Bernoulli numbers; Hypergeometric Bernoulli numbers; Hypergeometric functions; Kummer's congruence; Determinants; Recurrence relations; Continued fractions; Convergents

\section{Introduction}

Denote ${ }_{1} F_{1}(a ; b ; z)$ be the confluent hypergeometric function defined by

$$
{ }_{1} F_{1}(a ; b ; z)=\sum_{n=0}^{\infty} \frac{(a)^{(n)}}{(b)^{(n)}} \frac{z^{n}}{n !}
$$

with the rising factorial $(x)^{(n)}=x(x+1) \cdots(x+n-1)(n \geq 1)$ and $(x)^{(0)}=1$. For $N \geq 1$, define hypergeometric Bernoulli numbers $B_{N, n}[13-16,18]$ by

$$
\frac{1}{{ }_{1} F_{1}(1 ; N+1 ; x)}=\frac{x^{N} / N !}{e^{x}-\sum_{n=0}^{N-1} x^{n} / n !}=\sum_{n=0}^{\infty} B_{N, n} \frac{x^{n}}{n !} .
$$

When $N=1, B_{1, n}=B_{n}$ are the classical Bernoulli numbers, defined by

$$
\frac{x}{e^{x}-1}=\sum_{n=0}^{\infty} B_{n} \frac{x^{n}}{n !}
$$

(c) The Author(s) 2019. This article is distributed under the terms of the Creative Commons Attribution 4.0 International License (http://creativecommons.org/licenses/by/4.0/), which permits unrestricted use, distribution, and reproduction in any medium, provided you give appropriate credit to the original author(s) and the source, provide a link to the Creative Commons license, and indicate if changes were made. 
In addition, define hypergeometric Bernoulli polynomials $B_{N, n}(z)$ [17] by the generating function

$$
\frac{e^{x z}}{{ }_{1} F_{1}(1 ; N+1 ; x)}=\sum_{n=0}^{\infty} B_{N, n}(z) \frac{x^{n}}{n !}
$$

It is well known [25] that

$$
\sum_{m=0}^{n}\left(\begin{array}{c}
n \\
m
\end{array}\right) B_{1, m}(x) B_{2, n-m}(y)=B_{2, n}(x+y)-\frac{n}{2} B_{1, n-1}(x+y) \quad(n \geq 1) .
$$

Many kinds of generalizations of the Bernoulli numbers have been considered by many authors. For example, poly-Bernoulli numbers [20], multiple Bernoulli numbers [5], Apostol-Bernoulli numbers [23], multi-poly-Bernoulli numbers [10, 11], degenerate Bernoulli numbers [6], various types of $q$-Bernoulli numbers [4], Bernoulli-Carlitz numbers [3]. One of the advantages of hypergeometric numbers is the natural extension of determinant expressions of the numbers.

In [22], some determinant expressions of hypergeometric Cauchy numbers are considered. In this paper, first we show a similar determinant expression of hypergeometric Bernoulli numbers and their generalizations. Then we study some relations between the hypergeometric Bernoulli numbers and the classical Bernoulli numbers which include Kummer's congruences. Furthermore, by applying Trudi's formula, we also have some different expressions and inversion relations. Finally, we determine explicit forms of the convergents of the continued fraction expansion of the generating function of the hypergeometric Bernoulli numbers, from which several identities for hypergeometric Bernoulli numbers are given.

\section{Some basic properties of hypergeometric Bernoulli numbers}

In this section, we list some basic properties of hypergeometric Bernoulli numbers.

Proposition 1 Let $N, n \geq 1$. We have

$$
\begin{aligned}
& \sum_{m=0}^{n}\left(\begin{array}{c}
N+n \\
m
\end{array}\right) B_{N, m}=0, \\
& B_{N, n}=n ! \sum_{k=1}^{n} \sum_{\substack{1_{1}+\cdots+i_{k}=n \\
i_{1}, \ldots, i_{k} \geq 1}} \frac{(-N !)^{k}}{\left(N+i_{1}\right) ! \cdots\left(N+i_{k}\right) !}, \\
& B_{N, n}=n ! \sum_{k=1}^{n}\left(\begin{array}{c}
n+1 \\
k+1
\end{array}\right) \sum_{\substack{i_{1}+\cdots+i_{k}=n \\
i_{1}, \ldots, i_{k} \geq 0}} \frac{(-N !)^{k}}{\left(N+i_{1}\right) ! \cdots\left(N+i_{k}\right) !} .
\end{aligned}
$$

Remark When $N=1$ in (3), we have a famous identity for Bernoulli numbers,

$$
\sum_{m=0}^{n}\left(\begin{array}{c}
n+1 \\
m
\end{array}\right) B_{m}=0 \quad(n \geq 1) .
$$


In some literature (e.g., [20]), the Bernoulli numbers $\mathfrak{B}_{n}$ are also defined by

$$
\frac{x}{1-e^{-x}}=\sum_{n=0}^{\infty} \mathfrak{B}_{n} \frac{x^{n}}{n !}
$$

Then we have

$$
\sum_{m=0}^{n}\left(\begin{array}{c}
n+1 \\
m
\end{array}\right) \mathfrak{B}_{m}=n+1 \quad(n \geq 1)
$$

(see, e.g., [1]). Notice that $B_{n}=(-1)^{n} \mathfrak{B}_{n}(n \geq 0)$.

By the identity (3), we get

$$
B_{N, n}=-\sum_{k=0}^{n-1} \frac{\left(\begin{array}{c}
N+n \\
k
\end{array}\right)}{\left(\begin{array}{c}
N+n \\
n
\end{array}\right)} B_{N, k}
$$

with $B_{N, 0}=1(N \geq 1)$. So, we have the exact forms of $B_{N, n}$ for small $n$ one by one. For larger or general $n$, we need the identity (4).

In a later section about Trudi's formula, we shall see a different expression of $B_{N, n}$ in Corollary 2. Further, an inversion expression can be obtained:

$$
\left(\begin{array}{c}
N+n \\
N
\end{array}\right)^{-1}=\sum_{k=1}^{n}(-1)^{k} \sum_{\substack{i_{1}+\cdots+i_{k}=n \\
i_{1}, \ldots, i_{k} \geq 1}}\left(\begin{array}{c}
n \\
i_{1}, \ldots, i_{k}
\end{array}\right) B_{N, i_{1}} \cdots B_{N, i_{k}}
$$

where $\left(\begin{array}{c}n \\ t_{1}, \ldots, t_{k}\end{array}\right)=\frac{n !}{t_{1} ! \cdots t_{k} !}$ are the multinomial coefficients.

Proof of Proposition 1. From the definition in (1), we have

$$
\begin{aligned}
\frac{x^{N}}{N !} & =\left(\sum_{i=0}^{\infty} \frac{x^{i+N}}{(i+N) !}\right)\left(\sum_{m=0}^{\infty} B_{N, m} \frac{x^{m}}{m !}\right) \\
& =x^{N} \sum_{n=0}^{\infty} \sum_{m=0}^{n} \frac{x^{n-m}}{(n-m+N) !} B_{N, m} \frac{x^{m}}{m !} \\
& =\sum_{n=0}^{\infty} \sum_{m=0}^{n} \frac{B_{N, m}}{(n-m+N) ! m !} x^{N+n} .
\end{aligned}
$$

Hence, for $n \geq 1$, we have (3).

The proof of (4) can be done by induction on $n$. Here, we shall prove directly by using the generating function. From the definition in (1), we have

$$
\begin{aligned}
\sum_{n=0}^{\infty} B_{N, n} \frac{x^{n}}{n !} & =\frac{x^{N} / N !}{e^{x}-\sum_{i=0}^{N-1} x^{i} / i !} \\
& =\frac{1}{\frac{N !}{x^{N}}\left(e^{x}-\sum_{i=0}^{N} x^{i} / i !\right)+1}=\sum_{k=0}^{\infty}\left(-\frac{N !}{x^{N}}\left(e^{x}-\sum_{i=0}^{N} \frac{x^{i}}{i !}\right)\right)^{k} .
\end{aligned}
$$

The identity (4) immediately follows by comparing coefficients of both sides. 
The identity (5) is a different expression of $B_{N, n}$ with binomial coefficients. The proof is similar to that of (4) and is omitted.

\section{Analog of Kummer's congruence}

In this section, we show an analog of Kummer's congruences in the classical Bernoulli numbers. Let $p$ be a prime number, and $v \geq 0$ be an integer. For a non-zero integer $m$, we define the $p$-adic order $\operatorname{ord}_{p}(m)(\in \mathbb{Z})$ of $m$ by $m=u p^{\operatorname{ord}_{p}(m)}$ where $u$ is an integer satisfying $p \nmid u$. If $m$ and $n$ are positive even integers with $m \equiv n\left(\bmod (p-1) p^{v}\right)$ and $m, n \neq \equiv(\bmod p-1)$, then we have

$$
\left(1-p^{m-1}\right) \frac{B_{m}}{m} \equiv\left(1-p^{n-1}\right) \frac{B_{n}}{n} \quad\left(\bmod p^{v+1}\right)
$$

and this is called Kummer's congruence [30, Corollary 5.14]. We get the similar congruence for hypergeometric Bernoulli numbers $B_{N, n}$ for a special case that $N$ is $p$-adically close enough to 1 , that is, $\operatorname{ord}_{p}(N-1)$ is large enough compared to $v$ and the order of $\prod_{k=0}^{\max \{m, n\}}(1+k)$ !.

We need the following lemma in order to prove the main result.

Lemma 1 Let $p$ be a prime number. For $N \geq 1$ and $n \geq 0$, we have

$$
\prod_{k=0}^{n} \frac{(N+k) !}{N !} B_{N, n} \equiv \prod_{k=0}^{n}(1+k) ! B_{n} \quad\left(\bmod p^{t}\right),
$$

where $t=\operatorname{ord}_{p}(N-1)$.

Proof In the case $n=0$, the assertion is trivial. Assume that the result is true up to $n-1$. By Proposition 1, we have

$$
\begin{aligned}
\prod_{k=0}^{n} \frac{(N+k) !}{N !} B_{N, n} & =n ! \prod_{k=0}^{n-1} \frac{(N+k) !}{N !} \times\left\{-\sum_{m=0}^{n-1}\left(\begin{array}{c}
N+n \\
m
\end{array}\right) B_{N, m}\right\} \\
& =-\sum_{m=0}^{n-1} n !\left(\begin{array}{c}
N+n \\
m
\end{array}\right) \prod_{k=m+1}^{n-1} \frac{(N+k) !}{N !} \times \prod_{k=0}^{m} \frac{(N+k) !}{N !} B_{N, m} \\
& \equiv-\sum_{m=0}^{n-1} n !\left(\begin{array}{c}
N+n \\
m
\end{array}\right) \prod_{k=m+1}^{n-1} \frac{(N+k) !}{N !} \times \prod_{k=0}^{m}(1+k) ! B_{m} \quad\left(\bmod p^{t}\right) \\
& \equiv-\prod_{k=0}^{n-1}(1+k) ! \times n ! \sum_{m=0}^{n-1}\left(\begin{array}{c}
n+1 \\
m
\end{array}\right) B_{m} \quad\left(\bmod p^{t}\right) \\
& =\prod_{k=0}^{n}(1+k) ! B_{n} \quad\left(\bmod p^{t}\right) .
\end{aligned}
$$

From this lemma, we have the following corollary.

Corollary 1 Let $p$ be a prime number, and $N, n \geq 1, v \geq 0$ be integers with $n \neq 0$ $(\bmod p-1) \cdot$ If $\operatorname{ord}_{p}(N-1) \geq v+1+\operatorname{ord}_{p}\left(\prod_{k=0}^{n}(1+k) !\right)+\operatorname{ord}_{p}(n)$, then we have

$$
\frac{B_{N, n}}{n} \equiv \frac{B_{n}}{n} \quad\left(\bmod p^{v+1}\right) \text {. }
$$


Furthermore, by using (6), we have the following proposition.

Proposition 2 Let $p$ be a prime number, and $v \geq 0$ be an integer. If $m$ and $n$ are positive even integers with $m \geq n, m \equiv n\left(\bmod (p-1) p^{v}\right)$ and $m, n \neq \equiv 0(\bmod (p-1))$, and $\operatorname{ord}_{p}(N-$ $1) \geq v+1+\operatorname{ord}_{p}\left(\prod_{k=0}^{m}(1+k) !\right)+\max \left\{\operatorname{ord}_{p}(m), \operatorname{ord}_{p}(n)\right\}$, then we have

$$
\left(1-p^{m-1}\right) \frac{B_{N, m}}{m} \equiv\left(1-p^{n-1}\right) \frac{B_{N, n}}{n} \quad\left(\bmod p^{v+1}\right) .
$$

Note that $\operatorname{ord}_{p}\left(\prod_{k=0}^{m}(1+k) !\right)+\max \left\{\operatorname{ord}_{p}(m), \operatorname{ord}_{p}(m)\right\}=0$ if $p>m+1$ in Proposition 2.

Example 1 Consider the case $p=5, m=6, n=2$. For any integer $N$ satisfying

$$
\operatorname{ord}_{5}(N-1) \geq 1+\operatorname{ord}_{5}\left(\prod_{k=0}^{6}(1+k) !\right)=4
$$

we have

$$
\frac{B_{N, 6}}{6} \equiv \frac{B_{N, 2}}{2} \equiv 3(\bmod 5)
$$

Example 2 Consider the case $p=5, m=22, n=2$. For any integer $N$ satisfying

$$
\operatorname{ord}_{5}(N-1) \geq 2+\operatorname{ord}_{5}\left(\prod_{k=0}^{22}(1+k) !\right)=48,
$$

we have

$$
\left(1-5^{21}\right) \frac{B_{N, 22}}{22} \equiv(1-5) \frac{B_{N, 2}}{2} \equiv 8 \quad(\bmod 25) .
$$

\section{Determinant expressions}

We give a determinant expression of hypergeometric Bernoulli numbers.

Theorem 1 For $N, n \geq 1$, we have

$$
B_{N, n}=(-1)^{n} n !\left|\begin{array}{ccccc}
\frac{N !}{(N+1) !} & 1 & & & \\
\frac{N !}{(N+2) !} & \frac{N !}{(N+1) !} & & & \\
\vdots & \vdots & \ddots & 1 & \\
\frac{N !}{(N+n-1) !} & \frac{N !}{(N+n-2) !} & \cdots & \frac{N !}{(N+1) !} & 1 \\
\frac{N !}{(N+n) !} & \frac{N !}{(N+n-1) !} & \cdots & \frac{N !}{(N+2) !} & \frac{N !}{(N+1) !}
\end{array}\right| .
$$

Remark When $N=1$, we have a determinant expression of Bernoulli numbers [7, p. 53]:

$$
B_{n}=(-1)^{n} n !\left|\begin{array}{ccccc}
\frac{1}{2 !} & 1 & & & \\
\frac{1}{3 !} & \frac{1}{2 !} & & & \\
\vdots & \vdots & \ddots & 1 & \\
\frac{1}{n !} & \frac{1}{(n-1) !} & \cdots & \frac{1}{2 !} & 1 \\
\frac{1}{(n+1) !} & \frac{1}{n !} & \cdots & \frac{1}{3 !} & \frac{1}{2 !}
\end{array}\right| .
$$

Proof of Theorem 1 This theorem is a special case of Theorem 2. 
5 A relation between $B_{N, n}$ and $B_{N-1, n}$

In this section, we show the following relation between $B_{N, n}$ and $B_{N-1, n}$.

Proposition 3 For $N \geq 2$ and $n \geq 1$, we have

$$
\begin{aligned}
B_{N, n}= & \frac{N}{N+n}\left\{\sum_{m=0}^{n-1} \sum_{1 \leq i_{m}<\cdots<i_{1}<i_{0}=n} B_{N-1, i_{m}}\right. \\
& \left.\times \prod_{k=1}^{m} B_{N-1, i_{k-1}-i_{k}+1}\left(\begin{array}{c}
i_{k-1} \\
i_{k-1}-i_{k}+1
\end{array}\right) \frac{N}{N+i_{k}}\right\} .
\end{aligned}
$$

\section{Example 3}

(i) $B_{N, 1}=\frac{N}{N+1} B_{N-1,1}$

(ii) $B_{N, 2}=\frac{N}{N+2}\left\{B_{N-1,2}+\frac{N}{N+1} B_{N-1,1} B_{N-1,2}\right\}$,

(iii)

$$
\begin{aligned}
B_{N, 3}= & \frac{N}{N+3}\left\{B_{N-1,3}+\frac{N}{N+1} B_{N-1,1} B_{N-1,3}+\frac{3 N}{N+2} B_{N-1,2}^{2}\right. \\
& \left.+\frac{3 N^{2}}{(N+1)(N+2)} B_{N-1,1} B_{N-1,2}^{2}\right\} .
\end{aligned}
$$

By using Proposition 3 for $N=2$ and $B_{n}=0$ for odd $n \geq 3$, the numbers $B_{2, n}(0 \leq n \leq 4)$ are explicitly given by the classical Bernoulli numbers $B_{n}$ (cf. [15, \$9]),

$$
\begin{aligned}
& B_{2,0}=B_{1}(=1), \\
& B_{2,1}=\frac{2}{3} B_{1}\left(=-\frac{1}{3}\right), \\
& B_{2,2}=\frac{1}{2} B_{2}+\frac{1}{3} B_{1} B_{2}\left(=\frac{1}{2 \times 3^{2}}\right), \\
& B_{2,3}=\frac{3}{5} B_{2}^{2}+\frac{2}{5} B_{1} B_{2}^{2}\left(=\frac{1}{2 \times 3^{2} \times 5}\right), \\
& B_{2,4}=\frac{1}{3} B_{4}+\frac{2}{9} B_{1} B_{4}+\frac{6}{5} B_{2}^{3}+\frac{4}{5} B_{1} B_{2}^{3}\left(=-\frac{1}{2 \times 3^{3} \times 5}\right) .
\end{aligned}
$$

Lemma 2 For $N \geq 2$ and $n \geq 1$, we have

$$
B_{N, n}=\frac{N}{N+n}\left\{B_{N-1, n}+\sum_{m=1}^{n-1}\left(\begin{array}{c}
n \\
n-m+1
\end{array}\right) B_{N, m} B_{N-1, n-m+1}\right\} .
$$

Proof From the derivative of (1), we have

$$
\sum_{k=0}^{\infty} \frac{B_{N-1, k}}{k !} x^{k}=\left(\sum_{k=0}^{\infty} \frac{B_{N, k+1}}{k !} x^{k}\right)\left(1-\sum_{k=0}^{\infty} \frac{B_{N-1, k}}{k !} x^{k}\right)+\sum_{k=0}^{\infty} \frac{B_{N, k}}{k !} x^{k} .
$$

By $B_{N-1,0}=1$, we have

$$
\sum_{k=0}^{\infty} \frac{B_{N-1, k}}{k !} x^{k}=-\sum_{k=0}^{\infty} \sum_{\ell=0}^{\infty} B_{N, k+1} B_{N-1, \ell+1} \frac{x^{k+\ell+1}}{k !(\ell+1) !}+\sum_{k=0}^{\infty} \frac{B_{N, k}}{k !} x^{k}
$$




$$
\begin{aligned}
& =-\sum_{n=1}^{\infty} \sum_{\ell=0}^{n-1} B_{N, n-\ell} B_{N-1, \ell+1}\left(\begin{array}{c}
n \\
\ell+1
\end{array}\right) \frac{x^{n}}{n !}+\sum_{k=0}^{\infty} \frac{B_{N, k}}{k !} x^{k} \\
& =\sum_{n=1}^{\infty}\left\{B_{N, n}-\sum_{\ell=0}^{n-1}\left(\begin{array}{c}
n \\
\ell+1
\end{array}\right) B_{N, n-\ell} B_{N-1, \ell+1}\right\} \frac{x^{n}}{n !}+B_{N, 0} .
\end{aligned}
$$

Therefore, we have

$$
B_{N-1, n}=B_{N, n}-\sum_{\ell=0}^{n-1}\left(\begin{array}{c}
n \\
\ell+1
\end{array}\right) B_{N, n-\ell} B_{N-1, \ell+1},
$$

for $n \geq 1$. By $B_{N-1,1}=-\frac{1}{N}$, the assertion follows.

Proof of Proposition 3 We give the proof by induction for $n$. In the case $n=1$, the assertion means $B_{N, 1}=\frac{N}{N+1} B_{N-1,1}$, and this equality follows from $B_{N, 1}=-\frac{1}{N+1}$ and $B_{N-1,1}=-\frac{1}{N}$. Assume that the assertion holds up to $n-1$. By Lemma 2, we have

$$
\begin{aligned}
& B_{N, n}=\frac{N}{N+n}\left\{B_{N-1, n}+\sum_{i_{1}=1}^{n-1}\left(\begin{array}{c}
n \\
n-i_{1}+1
\end{array}\right) B_{N-1, n-i_{1}+1} \frac{N}{N+i_{1}}\right. \\
& \left.\times\left(\sum_{m=0}^{i_{1}-1} \sum_{1 \leq i_{m+1}<\cdots<i_{2}<i_{1}} B_{N-1, i_{m+1}} \prod_{k=2}^{m+1} B_{N-1, i_{k-1}-i_{k}+1}\left(\begin{array}{c}
i_{k-1} \\
i_{k-1}-i_{k}+1
\end{array}\right) \frac{N}{N+i_{k}}\right)\right\} \\
& =\frac{N}{N+n}\left\{B_{N-1, n}+\sum_{i_{1}=1}^{n-1} \sum_{m=0}^{i_{1}-1} \sum_{1 \leq i_{m+1}<\cdots<i_{2}<i_{1}} B_{N-1, i_{m+1}}\right. \\
& \left.\times \prod_{k=1}^{m+1} B_{N-1, i_{k-1}-i_{k}+1}\left(\begin{array}{c}
i_{k-1} \\
i_{k-1}-i_{k}+1
\end{array}\right) \frac{N}{N+i_{k}}\right\} \\
& =\frac{N}{N+n}\left\{B_{N-1, n}+\sum_{i_{1}=1}^{n-1} \sum_{\ell=1}^{i_{1}} \sum_{1 \leq i_{\ell}<\cdots<i_{2}<i_{1}} B_{N-1, i_{\ell}}\right. \\
& \left.\left.\times \prod_{k=1}^{\ell} B_{N-1, i_{k-1}-i_{k}+1}\left(\begin{array}{c}
i_{k-1} \\
i_{k-1}-i_{k}+1
\end{array}\right) \frac{N}{N+i_{k}}\right)\right\} \\
& =\frac{N}{N+n}\left\{B_{N-1, n}+\sum_{m=1}^{n-1} \sum_{1 \leq i_{m}<\cdots<i_{1} \leq n-1} B_{N-1, i_{m}}\right. \\
& \left.\times \prod_{k=1}^{m} B_{N-1, i_{k-1}-i_{k}+1}\left(\begin{array}{c}
i_{k-1} \\
i_{k-1}-i_{k}+1
\end{array}\right) \frac{N}{N+i_{k}}\right\} \\
& =\frac{N}{N+n}\left\{\sum_{m=0}^{n-1} \sum_{1 \leq i_{m}<\cdots<i_{1}<i_{0}=n} B_{N-1, i_{m}}\right. \\
& \left.\times \prod_{k=1}^{m} B_{N-1, i_{k-1}-i_{k}+1}\left(\begin{array}{c}
i_{k-1} \\
i_{k-1}-i_{k}+1
\end{array}\right) \frac{N}{N+i_{k}}\right\} .
\end{aligned}
$$

\section{Multiple hypergeometric Bernoulli numbers}

In this section, we define a more general hypergeometric Bernoulli number and give the properties. 
For positive integers $N$ and $r$, define the higher order hypergeometric Bernoulli numbers $B_{N, n}^{(r)}[18,26]$ by the generating function

$$
\frac{1}{{ }_{1} F_{1}(1 ; N+1 ; x)^{r}}=\left(\frac{x^{N} / N !}{e^{x}-\sum_{n=0}^{N-1} x^{n} / n !}\right)^{r}=\sum_{n=0}^{\infty} B_{N, n}^{(r)} \frac{x^{n}}{n !} .
$$

The higher order hypergeometric Bernoulli polynomials $B_{N, n}^{(r)}(x)$ are studied in [17], we have $B_{N, n}^{(r)}=B_{N, n}^{(r)}(0)$.

From the definition (8), we have

$$
\begin{aligned}
\left(\frac{x^{N}}{N !}\right)^{r} & =\left(\sum_{i=0}^{\infty} \frac{x^{i+N}}{(i+N) !}\right)^{r}\left(\sum_{m=0}^{\infty} B_{N, m}^{(r)} \frac{x^{m}}{m !}\right) \\
& =x^{r N}\left(\sum_{l=0}^{\infty} \sum_{\substack{i_{1}+\cdots+i_{r}=l \\
i_{1}, \ldots, i_{r} \geq 0}} \frac{l !}{\left(N+i_{1}\right) ! \cdots\left(N+i_{r}\right) !} \frac{x^{l}}{l !}\right)\left(\sum_{m=0}^{\infty} B_{N, m}^{(r)} \frac{x^{m}}{m !}\right) \\
& =x^{r N} \sum_{n=0}^{\infty} \sum_{m=0}^{n} \sum_{\substack{i_{1}+\cdots+i_{r}=n-m \\
i_{1}, \ldots, i_{r} \geq 0}}^{n}\left(\begin{array}{c}
n \\
m
\end{array}\right) \frac{(n-m) !}{\left(N+i_{1}\right) ! \cdots\left(N+i_{r}\right) !} B_{N, m}^{(r)} \frac{x^{n}}{n !} .
\end{aligned}
$$

Hence, as a generalization of Proposition 1 (3), for $n \geq 1$, we have the following.

\section{Proposition 4 We have}

$$
\sum_{m=0}^{n} \sum_{\substack{i_{1}+\cdots+i_{r}=n-m \\ i_{1}, \ldots, i_{r} \geq 0}} \frac{B_{N, m}^{(r)}}{m !\left(N+i_{1}\right) ! \cdots\left(N+i_{r}\right) !}=0
$$

By using Proposition 4 or

$$
B_{N, n}^{(r)}=-n !(N !)^{r} \sum_{m=0}^{n-1} \sum_{\substack{i_{1}+\cdots+i_{r}=n-m \\ i_{1}, \ldots, i_{r} \geq 0}} \frac{B_{N, m}^{(r)}}{m !\left(N+i_{1}\right) ! \cdots\left(N+i_{r}\right) !}
$$

with $B_{N, 0}^{(r)}=1(N \geq 1)$, some values of $B_{N, n}^{(r)}(0 \leq n \leq 4)$ are explicitly given by the following:

$$
\begin{aligned}
B_{N, 0}^{(r)=} & 1, \\
B_{N, 1}^{(r)}= & -\frac{r}{N+1}, \\
B_{N, 2}^{(r)}= & \frac{2 r}{(N+1)^{2}(N+2)}\left(-(N+1)+\frac{r+1}{2}(N+2)\right), \\
B_{N, 3}^{(r)}= & \frac{3 ! r}{(N+1)^{3}(N+2)(N+3)}\left(-(N+1)^{2}+(r+1)(N+1)(N+3)\right. \\
& \left.-\frac{(r+1)(r+2)}{6}(N+2)(N+3)\right) \\
B_{N, 4}^{(r)}= & \frac{4 ! r}{(N+1)^{4}(N+2)^{2}(N+3)(N+4)}\left(-(N+1)^{3}(N+2)\right.
\end{aligned}
$$




$$
\begin{aligned}
& +(r+1)(N+1)^{2}(N+2)(N+4)+\frac{r+1}{2}(N+1)^{2}(N+3)(N+4) \\
& -\frac{(r+1)(r+2)}{2}(N+1)(N+2)(N+3)(N+4) \\
& \left.+\frac{(r+1)(r+2)(r+3)}{4 !}(N+2)^{2}(N+3)(N+4)\right) .
\end{aligned}
$$

As a generalization of Proposition 1 (4), we have an explicit expression of $B_{N, n}^{(r)}$.

Proposition 5 For $N, n \geq 1$, we have

$$
B_{N, n}^{(r)}=n ! \sum_{k=1}^{n}(-1)^{k} \sum_{\substack{e_{1}+\cdots+e_{k}=n \\ e_{1}, \ldots, e_{k} \geq 1}} M_{r}\left(e_{1}\right) \cdots M_{r}\left(e_{k}\right)
$$

where

$$
M_{r}(e)=\sum_{\substack{i_{1}+\cdots+i_{r}=e \\ i_{1}, \ldots, i_{r} \geq 0}} \frac{(N !)^{r}}{\left(N+i_{1}\right) ! \cdots\left(N+i_{r}\right) !} .
$$

We shall introduce the Hasse-Teichmüller derivative in order to prove Proposition 5 easily. Let $\mathbb{F}$ be a field of any characteristic, $\mathbb{F}[[z]]$ the ring of formal power series in one variable $z$, and $\mathbb{F}((z))$ the field of Laurent series in $z$. Let $n$ be a nonnegative integer. We define the Hasse-Teichmüller derivative $H^{(n)}$ of order $n$ by

$$
H^{(n)}\left(\sum_{m=R}^{\infty} c_{m} z^{m}\right)=\sum_{m=R}^{\infty} c_{m}\left(\begin{array}{l}
m \\
n
\end{array}\right) z^{m-n}
$$

for $\sum_{m=R}^{\infty} c_{m} z^{m} \in \mathbb{F}((z))$, where $R$ is an integer and $c_{m} \in \mathbb{F}$ for any $m \geq R$. Note that $\left(\begin{array}{c}m \\ n\end{array}\right)=0$ if $m<n$.

The Hasse-Teichmüller derivatives satisfy the product rule [27], the quotient rule [8] and the chain rule [12]. One of the product rules can be described as follows.

Lemma 3 For $f_{i} \in \mathbb{F}[[z]](i=1, \ldots, k)$ with $k \geq 2$ and for $n \geq 1$, we have

$$
H^{(n)}\left(f_{1} \cdots f_{k}\right)=\sum_{\substack{i_{1}+\cdots+i_{k}=n \\ i_{1}, \ldots, i_{k} \geq 0}} H^{\left(i_{1}\right)}\left(f_{1}\right) \cdots H^{\left(i_{k}\right)}\left(f_{k}\right)
$$

The quotient rules can be described as follows.

Lemma 4 For $f \in \mathbb{F}[[z]] \backslash\{0\}$ and $n \geq 1$, we have

$$
\begin{aligned}
H^{(n)}\left(\frac{1}{f}\right) & =\sum_{k=1}^{n} \frac{(-1)^{k}}{f^{k+1}} \sum_{\substack{i_{1}+\cdots+i_{k}=n \\
i_{1}, \ldots, i_{k} \geq 1}} H^{\left(i_{1}\right)}(f) \cdots H^{\left(i_{k}\right)}(f) \\
& =\sum_{k=1}^{n}\left(\begin{array}{l}
n+1 \\
k+1
\end{array}\right) \frac{(-1)^{k}}{f^{k+1}} \sum_{\substack{i_{1}+\cdots+i_{k}=n \\
i_{1}, \ldots, i_{k} \geq 0}} H^{\left(i_{1}\right)}(f) \cdots H^{\left(i_{k}\right)}(f) .
\end{aligned}
$$


Proof of Proposition 5 Put $h(x)=(f(x))^{r}$, where

$$
f(x)=\frac{\sum_{i=N}^{\infty} \frac{x^{i}}{i !}}{\frac{x^{N}}{N !}}=\sum_{j=0}^{\infty} \frac{N !}{(N+j) !} x^{j}
$$

Since

$$
\begin{aligned}
\left.H^{(i)}(f)\right|_{x=0} & =\left.\sum_{j=i}^{\infty} \frac{N !}{(N+j) !}\left(\begin{array}{l}
j \\
i
\end{array}\right) x^{j-i}\right|_{x=0} \\
& =\frac{N !}{(N+i) !}
\end{aligned}
$$

by the product rule of the Hasse-Teichmüller derivative in Lemma 3, we get

$$
\begin{aligned}
\left.H^{(e)}(h)\right|_{x=0} & =\left.\left.\sum_{\substack{i_{1}+\cdots+i_{r}=e \\
i_{1}, \ldots, i_{r} \geq 0}} H^{\left(i_{1}\right)}(f)\right|_{x=0} \cdots H^{\left(i_{r}\right)}(f)\right|_{x=0} \\
& =\sum_{\substack{i_{1}+\cdots+i_{r}=e \\
i_{1}, \ldots, i_{r} \geq 0}} \frac{N !}{\left(N+i_{1}\right) !} \cdots \frac{N !}{\left(N+i_{r}\right) !}=M_{r}(e) .
\end{aligned}
$$

Hence, by the quotient rule of the Hasse-Teichmüller derivative in Lemma 4 (11), we have

$$
\begin{aligned}
\frac{B_{N, n}^{(r)}}{n !} & =\left.\left.\left.\sum_{k=1}^{n} \frac{(-1)^{k}}{h^{k+1}}\right|_{\substack { x=0 \\
\begin{subarray}{c}{e_{1}+\cdots+e_{k}=n \\
e_{1}, \ldots, e_{k} \geq 1{ x = 0 \\
\begin{subarray} { c } { e _ { 1 } + \cdots + e _ { k } = n \\
e _ { 1 } , \ldots , e _ { k } \geq 1 } }\end{subarray}} H^{\left(e_{1}\right)}(h)\right|_{x=0} \cdots H^{\left(e_{k}\right)}(h)\right|_{x=0} \\
& =\sum_{k=1}^{n}(-1)^{k} \sum_{\substack{e_{1}+\cdots+e_{k}=n \\
e_{1}, \ldots, e_{k} \geq 1}} M_{r}\left(e_{1}\right) \cdots M_{r}\left(e_{k}\right) .
\end{aligned}
$$

Now, we can also show a determinant expression of $B_{N, n}^{(r)}$.

Theorem 2 For $N, n \geq 1$, we have

$$
B_{N, n}^{(r)}=(-1)^{n} n !\left|\begin{array}{ccccc}
M_{r}(1) & 1 & & & \\
M_{r}(2) & M_{r}(1) & & & \\
\vdots & \vdots & \ddots & 1 & \\
M_{r}(n-1) & M_{r}(n-2) & \ldots & M_{r}(1) & 1 \\
M_{r}(n) & M_{r}(n-1) & \cdots & M_{r}(2) & M_{r}(1)
\end{array}\right|
$$

where $M_{r}(e)$ are given in (10).

Remark When $r=1$ in Theorem 2, we have the result in Theorem 1 . 
Proof For simplicity, put $A_{N, n}^{(r)}=(-1)^{n} B_{N, n}^{(r)} / n !$. Then we shall prove that for any $n \geq 1$,

$$
A_{N, n}^{(r)}=\left|\begin{array}{ccccc}
M_{r}(1) & 1 & & & \\
M_{r}(2) & M_{r}(1) & & & \\
\vdots & \vdots & \ddots & 1 & \\
M_{r}(n-1) & M_{r}(n-2) & \cdots & M_{r}(1) & 1 \\
M_{r}(n) & M_{r}(n-1) & \cdots & M_{r}(2) & M_{r}(1)
\end{array}\right| .
$$

When $n=1$, (13) is valid because

$$
M_{r}(1)=\frac{r(N !)^{r}}{(N !)^{r-1}(N+1) !}=\frac{r}{N+1}=A_{N, 1}^{(r)} .
$$

Assume that (13) is valid up to $n-1$. Notice that by (9), we have

$$
A_{N, n}^{(r)}=\sum_{l=1}^{n}(-1)^{l-1} A_{N, n-l}^{(r)} M_{r}(l) .
$$

Thus, by expanding the first row of the right-hand side of (13), it is equal to

$$
\begin{aligned}
& M_{r}(1) A_{N, n-1}^{(r)}-\left|\begin{array}{ccccc}
M_{r}(2) & 1 & & & \\
M_{r}(3) & M_{r}(1) & & & \\
\vdots & \vdots & \ddots & 1 & \\
M_{r}(n-1) & M_{r}(n-3) & \cdots & M_{r}(1) & 1 \\
M_{r}(n) & M_{r}(n-2) & \cdots & M_{r}(2) & M_{r}(1)
\end{array}\right| \\
& =M_{r}(1) A_{N, n-1}^{(r)}-M_{r}(2) A_{N, n-2}^{(r)} \\
& +\left|\begin{array}{ccccc}
M_{r}(3) & 1 & & & \\
M_{r}(4) & M_{r}(1) & & & \\
\vdots & \vdots & \ddots & 1 & \\
M_{r}(n-1) & M_{r}(n-4) & \cdots & M_{r}(1) & 1 \\
M_{r}(n) & M_{r}(n-3) & \cdots & M_{r}(2) & M_{r}(1)
\end{array}\right| \\
& =M_{r}(1) A_{N, n-1}^{(r)}-M_{r}(2) A_{N, n-2}^{(r)}+\cdots+(-1)^{n-2}\left|\begin{array}{cc}
M_{r}(n-1) & 1 \\
M_{r}(n) & M_{r}(1)
\end{array}\right| \\
& =\sum_{l=1}^{n}(-1)^{l-1} M_{r}(l) A_{N, n-l}^{(r)}=A_{N, n}^{(r)} .
\end{aligned}
$$

Note that $A_{N, 1}^{(r)}=M_{r}(1)$ and $A_{N, 0}^{(r)}=1$.

\section{A relation between $B_{N, n}^{(r)}$ and $B_{N, n}$}

In this section, we show the following relation between $B_{N, n}^{(r)}$ and $B_{N, n}$.

Lemma 5 For $r, N \geq 1$ and $n \geq 0$, we have

$$
B_{N, n}^{(r)}=\sum_{\substack{n_{1}, \ldots, n_{r} \geq 0 \\ n_{1}+\cdots+n_{r}=n}} \frac{n !}{n_{1} ! \cdots n_{r} !} B_{N, n_{1}} \cdots B_{N, n_{r}} .
$$


Proof From the definition (1) and (8), we get the assertion.

We give some examples for Lemma 5 . Note that $B_{N, n}=B_{N, n}^{(1)}$ by the definition.

\section{Example 4}

(i) $B_{N, 0}^{(r)}=B_{N, 0}^{r}(r \geq 1)$,

(ii) $B_{N, 1}^{(r)}=r B_{N, 1} B_{N, 0}^{r-1}(r \geq 1)$,

(iii)

$$
\begin{aligned}
B_{N, 2}^{(r)} & =\sum_{\substack{n_{1}, \ldots, n_{r} \geq 0 \\
n_{1}+\cdots+n_{r}=2}} \frac{2}{n_{1} ! \cdots n_{r} !} B_{N, n_{1}} \cdots B_{N, n_{r}} \\
& =\sum_{\substack{n_{1}, \ldots, n_{r} \in\{0,2\} \\
n_{1}+\cdots+n_{r}=2}} \frac{2}{n_{1} ! \cdots n_{r} !} B_{N, n_{1}} \cdots B_{N, n_{r}}+\sum_{\substack{n_{1}, \ldots, n_{r} \in\{0,1\} \\
n_{1}+\cdots+n_{r}=2}} \frac{2}{n_{1} ! \cdots n_{r} !} B_{N, n_{1}} \cdots B_{N, n_{r}} \\
& =r B_{N, 2} B_{N, 0}^{N-1}+r(r-1) B_{N, 1}^{2} B_{N, 0}^{r-2} \quad(r \geq 2) .
\end{aligned}
$$

\section{Applications by the Trudi's formula and inversion expressions}

We can obtain different explicit expressions for the numbers $B_{N, n}^{(r)}, B_{N, n}$ and $B_{n}$ by using the Trudi's formula. We also show some inversion formulas. The following relation is known as Trudi's formula [24, Vol. 3, p. 214], [28] and the case $a_{0}=1$ of this formula is known as Brioschi's formula [2], [24, Vol. 3, pp. 208-209].

Lemma 6 For a positive integer m, we have

$$
\begin{aligned}
& \left|\begin{array}{ccccc}
a_{1} & a_{2} & \cdots & \cdots & a_{m} \\
a_{0} & a_{1} & \ddots & & \vdots \\
& \ddots & \ddots & \ddots & \vdots \\
& & \ddots & a_{1} & a_{2} \\
a_{0} & a_{1}
\end{array}\right| \\
& \quad=\sum_{t_{1}+2 t_{2}+\cdots+m t_{m}=m}\left(\begin{array}{c}
t_{1}+\cdots+t_{m} \\
t_{1}, \ldots, t_{m}
\end{array}\right)\left(-a_{0}\right)^{m-t_{1}-\cdots-t_{m}} a_{1}^{t_{1}} a_{2}^{t_{2}} \cdots a_{m}^{t_{m}},
\end{aligned}
$$

where $\left(\begin{array}{c}t_{1}+\cdots+t_{m} \\ t_{1}, \ldots, t_{m}\end{array}\right)=\frac{\left(t_{1}+\cdots+t_{m}\right) !}{t_{1} ! \cdots t_{m} !}$ are the multinomial coefficients.

In addition, there exists the following inversion formula (see, e.g., [21]), which is based upon the relation

$$
\sum_{k=0}^{n}(-1)^{n-k} \alpha_{k} R(n-k)=0 \quad(n \geq 1) .
$$


Lemma 7 If $\left\{\alpha_{n}\right\}_{n \geq 0}$ is a sequence defined by $\alpha_{0}=1$ and

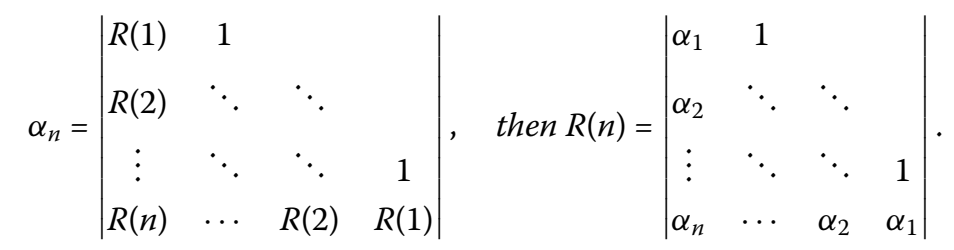

Moreover, if

$$
A=\left(\begin{array}{cccc}
1 & & & \\
\alpha_{1} & 1 & & \\
\vdots & \ddots & \ddots & \\
\alpha_{n} & \cdots & \alpha_{1} & 1
\end{array}\right) \text {, then } A^{-1}=\left(\begin{array}{cccc}
1 & & & \\
R(1) & 1 & & \\
\vdots & \ddots & \ddots & \\
R(n) & \cdots & R(1) & 1
\end{array}\right)
$$

From Trudi's formula, it is possible to give the combinatorial expression

$$
\alpha_{n}=\sum_{t_{1}+2 t_{2}+\cdots+n t_{n}=n}\left(\begin{array}{c}
t_{1}+\cdots+t_{n} \\
t_{1}, \ldots, t_{n}
\end{array}\right)(-1)^{n-t_{1}-\cdots-t_{n}} R(1)^{t_{1}} R(2)^{t_{2}} \cdots R(n)^{t_{n}} .
$$

By applying these lemmas to Theorem 2, we obtain an explicit expression for the generalized hypergeometric Bernoulli numbers $B_{N, n}^{(r)}$.

Theorem 3 For $n \geq 1$,

$$
B_{N, n}^{(r)}=n ! \sum_{t_{1}+2 t_{2}+\cdots+n t_{n}=n}\left(\begin{array}{c}
t_{1}+\cdots+t_{n} \\
t_{1}, \ldots, t_{n}
\end{array}\right)(-1)^{t_{1}+\cdots+t_{n}} M_{r}(1)^{t_{1}} M_{r}(2)^{t_{2}} \cdots M_{r}(n)^{t_{n}}
$$

where $M_{r}(e)$ are given in (10). Moreover,

$$
M_{r}(n)=\left|\begin{array}{cccc}
-\frac{B_{N, 1}^{(r)}}{1 !} & 1 & & \\
\frac{B_{N, 2}^{(r)}}{2 !} & \ddots & \ddots & \\
\vdots & \ddots & \ddots & 1 \\
\frac{(-1)^{n} B_{N, n}^{(r)}}{n !} & \ldots & \frac{B_{N, 2}^{(r)}}{2 !} & -\frac{B_{N, 1}^{(r)}}{1 !}
\end{array}\right|
$$

and

$$
\left(\begin{array}{ccccc}
1 & & & & \\
-\frac{B_{N, 1}^{(r)}}{(r)} & 1 & & & \\
\frac{B_{N, 2}^{(r)}}{2 !} & -\frac{B_{N, 1}^{(r)}}{1 !} & 1 & & \\
\vdots & & \ddots & \ddots & \\
\frac{(-1)^{n} B_{N, n}^{(r)}}{n !} & \ldots & \frac{B_{N, 2}^{(r)}}{2 !} & -\frac{B_{N, 1}^{(r)}}{1 !} & 1
\end{array}\right)^{-1}
$$




$$
=\left(\begin{array}{ccccc}
1 & & & & \\
M_{r}(1) & 1 & & & \\
M_{r}(2) & M_{r}(1) & 1 & & \\
\vdots & & \ddots & \ddots & \\
M_{r}(n) & \ldots & M_{r}(2) & M_{r}(1) & 1
\end{array}\right) .
$$

When $r=1$ in Theorem 3, we have an explicit expression for the numbers $B_{N, n}$.

Corollary 2 For $n \geq 1$,

$$
\begin{aligned}
B_{N, n}= & n ! \sum_{t_{1}+2 t_{2}+\cdots+n t_{n}=n}\left(\begin{array}{c}
t_{1}+\cdots+t_{n} \\
t_{1}, \ldots, t_{n}
\end{array}\right)(-1)^{t_{1}+\cdots+t_{n}} \\
& \times\left(\frac{N !}{(N+1) !}\right)^{t_{1}}\left(\frac{N !}{(N+2) !}\right)^{t_{2}} \cdots\left(\frac{N !}{(N+n) !}\right)^{t_{n}}
\end{aligned}
$$

and

$$
\frac{N !}{(N+n) !}=\left|\begin{array}{cccc}
-\frac{B_{N, 1}}{1 !} & 1 & & \\
\frac{B_{N, 2}}{2 !} & \ddots & \ddots & \\
\vdots & \ddots & \ddots & 1 \\
\frac{(-1)^{n} B_{N, n}}{n !} & \ldots & \frac{B_{N, 2}}{2 !} & -\frac{B_{N, 1}}{1 !}
\end{array}\right| .
$$

When $r=N=1$ in Theorem 3, we have a different expression of the classical Bernoulli numbers.

Corollary 3 We have for $n \geq 1$,

$$
\begin{aligned}
B_{n}= & n ! \sum_{t_{1}+2 t_{2}+\cdots+n t_{n}=n}\left(\begin{array}{c}
t_{1}+\cdots+t_{n} \\
t_{1}, \ldots, t_{n}
\end{array}\right)(-1)^{t_{1}+\cdots+t_{n}} \\
& \times\left(\frac{1}{2 !}\right)^{t_{1}}\left(\frac{1}{3 !}\right)^{t_{2}} \cdots\left(\frac{1}{(n+1) !}\right)^{t_{n}}
\end{aligned}
$$

and

$$
\frac{1}{(n+1) !}=\left|\begin{array}{cccc}
-\frac{B_{1}}{1 !} & 1 & & \\
\frac{B_{2}}{2 !} & \ddots & \ddots & \\
\vdots & \ddots & \ddots & 1 \\
\frac{(-1)^{n} B_{n}}{n !} & \cdots & \frac{B_{2}}{2 !} & -\frac{B_{1}}{1 !}
\end{array}\right| .
$$

\section{Continued fractions of hypergeometric Bernoulli numbers}

In the final section, we show several identities for hypergeometric Bernoulli numbers which the convergents of the continued fraction expansion of the generating function of hypergeometric Bernoulli numbers entail.

In $[1,19]$ by studying the convergents of the continued fraction of

$$
\frac{x / 2}{\tanh x / 2}=\sum_{n=0}^{\infty} B_{2 n} \frac{x^{2 n}}{(2 n) !},
$$


some identities of Bernoulli numbers are obtained. In this section, the $n$th convergent of the generating function of hypergeometric Bernoulli numbers is explicitly given. As an application, we give some identities of hypergeometric Bernoulli numbers in terms of binomial coefficients.

The generating function on the left-hand side of (1) can be expanded as a continued fraction

$$
\frac{1}{{ }_{1} F_{1}(1 ; N+1 ; x)}=1-\frac{x}{N+1+\frac{x}{N+3+\frac{(N+1) x}{N+4-\frac{(N+2) x}{N+5+\ddots}}}}
$$

(cf. [29, (91.2)]). Its $n$th convergent $P_{n}(x) / Q_{n}(x)(n \geq 0)$ is given by the recurrence relation

$$
\begin{aligned}
& P_{n}(x)=a_{n}(x) P_{n-1}(x)+b_{n}(x) P_{n-2}(x) \quad(n \geq 2), \\
& Q_{n}(x)=a_{n}(x) Q_{n-1}(x)+b_{n}(x) Q_{n-2}(x) \quad(n \geq 2),
\end{aligned}
$$

with initial values

$$
\begin{array}{ll}
P_{0}(x)=1, & P_{1}(x)=(N+1)-x ; \\
Q_{0}(x)=1, & Q_{1}(x)=N+1,
\end{array}
$$

where, for $n \geq 1, a_{n}(x)=N+n, b_{2 n}(x)=n x$ and $b_{2 n+1}(x)=-(N+n) x$.

We have explicit expressions of both the numerator and the denominator of the $n$th convergent of (14).

Theorem 4 For $n \geq 1$, we have

$$
\begin{aligned}
& P_{2 n-1}(x)=\sum_{j=0}^{n}(-1)^{j}\left(\begin{array}{l}
n \\
j
\end{array}\right) \prod_{l=1}^{2 n-j-1}(N+l) \cdot x^{j}, \\
& P_{2 n}(x)=\sum_{j=0}^{n}(-1)^{j}\left(\begin{array}{l}
n \\
j
\end{array}\right) \prod_{l=1}^{2 n-j}(N+l) \cdot x^{j},
\end{aligned}
$$

and

$$
\begin{aligned}
& Q_{2 n-1}(x)=\sum_{j=0}^{n-1} \sum_{k=0}^{j}(-1)^{j-k}(2 n-j-1)_{k}\left(\begin{array}{c}
n-k-1 \\
j-k
\end{array}\right) \prod_{l=k+1}^{2 n-j-1}(N+l) \cdot x^{j}, \\
& Q_{2 n}(x)=\sum_{j=0}^{n} \sum_{k=0}^{j}(-1)^{j-k}(2 n-j)_{k}\left(\begin{array}{c}
n-k-1 \\
j-k
\end{array}\right) \prod_{l=k+1}^{2 n-j}(N+l) \cdot x^{j} .
\end{aligned}
$$


Remark Here we use the convenient values

$$
\left(\begin{array}{l}
n \\
k
\end{array}\right)=0 \quad(0 \leq n<k), \quad\left(\begin{array}{c}
-1 \\
0
\end{array}\right)=1,
$$

and recognize the empty product as 1 . Otherwise, we should write $Q_{2 n}(x)$ as

$$
Q_{2 n}(x)=\sum_{j=0}^{n-1} \sum_{k=0}^{j}(-1)^{j-k}(2 n-j)_{k}\left(\begin{array}{c}
n-k-1 \\
j-k
\end{array}\right) \prod_{l=k+1}^{2 n-j}(N+l) \cdot x^{j}+n ! x^{n} .
$$

If we use the unsigned Stirling numbers of the first kind, $\left[\begin{array}{l}n \\ k\end{array}\right]$, of which the generating function is given by

$$
\sum_{n=k}^{\infty}(-1)^{n-k}\left[\begin{array}{l}
n \\
k
\end{array}\right] \frac{z^{n}}{n !}=\frac{(\log (1+z))^{k}}{k !}
$$

$[9,(7.50)]$, we can express the products as

$$
\prod_{l=1}^{2 n-j-1}(N+l)=\sum_{i=1}^{2 n-j}\left[\begin{array}{c}
2 n-j \\
i
\end{array}\right] N^{i-1}
$$

or

$$
\prod_{l=k+1}^{2 n-j-1}(N+l)=\sum_{i=1}^{2 n-j-k}\left[\begin{array}{c}
2 n-j-k \\
i
\end{array}\right](N+k)^{i-1} .
$$

Proof of Theorem 4. The proof is done by induction on $n$. It is easy to see that for $n=0$, we have $P_{0}(x)=Q_{0}(x)=1$, and for $n=1$, we have $P_{1}(x)=(N+1)-x$ and $Q_{1}(x)=N+1$. Assume that the results hold up to $n-1(\geq 2)$. Then by using the recurrence relation in (16)

$$
\begin{aligned}
(N+2 n) P_{2 n-1}(x)+n P_{2 n-2}(x) \cdot x \\
=(N+2 n) \sum_{j=0}^{n}(-1)^{j}\left(\begin{array}{c}
n \\
j
\end{array}\right) \prod_{l=1}^{2 n-j-1}(N+l) \cdot x^{j} \\
+n \sum_{j=0}^{n-1}(-1)^{j}\left(\begin{array}{c}
n-1 \\
j
\end{array}\right) \prod_{l=1}^{2 n-j-2}(N+l) \cdot x^{j+1} \\
=(N+2 n) \prod_{l=1}^{2 n-1}(N+l) \\
+(N+2 n) \sum_{j=1}^{n}(-1)^{j}\left(\begin{array}{c}
n \\
j
\end{array}\right) \prod_{l=1}^{2 n-j-1}(N+l) \cdot x^{j} \\
\quad-n \sum_{j=1}^{n}(-1)^{j}\left(\begin{array}{c}
n-1 \\
j-1
\end{array}\right) \prod_{l=1}^{2 n-j-1}(N+l) \cdot x^{j} .
\end{aligned}
$$


Since

$$
(N+2 n)\left(\begin{array}{l}
n \\
j
\end{array}\right)-n\left(\begin{array}{c}
n-1 \\
j-1
\end{array}\right)=(N+2 n-j)\left(\begin{array}{l}
n \\
j
\end{array}\right)
$$

we get

$$
\begin{aligned}
(N & +2 n) P_{2 n-1}(x)+n P_{2 n-2}(x) \cdot x \\
& =\sum_{j=0}^{n}(-1)^{j}\left(\begin{array}{l}
n \\
j
\end{array}\right) \prod_{l=1}^{2 n-j}(N+l) \cdot x^{j} \\
& =P_{2 n} .
\end{aligned}
$$

Next,

$$
\begin{aligned}
(N+ & 2 n+1) P_{2 n}(x)-(N+n) P_{2 n-1}(x) \cdot x \\
= & (N+2 n+1) \sum_{j=0}^{n}(-1)^{j}\left(\begin{array}{c}
n \\
j
\end{array}\right) \prod_{l=1}^{2 n-j}(N+l) \cdot x^{j} \\
& -(N+n) \sum_{j=0}^{n}(-1)^{j}\left(\begin{array}{c}
n \\
j
\end{array}\right) \prod_{l=1}^{2 n-j-1}(N+l) \cdot x^{j+1} \\
= & (N+2 n+1) \prod_{l=1}^{2 n}(N+l) \\
& +(N+2 n+1) \sum_{j=1}^{n}(-1)^{j}\left(\begin{array}{c}
n \\
j
\end{array}\right) \prod_{l=1}^{2 n-j}(N+l) \cdot x^{j} \\
& +(N+n) \sum_{j=1}^{n}(-1)^{j}\left(\begin{array}{c}
n \\
j-1
\end{array}\right) \prod_{l=1}^{2 n-j}(N+l) \cdot x^{j} \\
& -(N+n)(-1)^{n} \prod_{l=1}^{n-1}(N+l) \cdot x^{n+1} \cdot
\end{aligned}
$$

Since

$$
(N+2 n+1)\left(\begin{array}{l}
n \\
j
\end{array}\right)+(N+n)\left(\begin{array}{c}
n \\
j-1
\end{array}\right)=(N+2 n-j+1)\left(\begin{array}{c}
n+1 \\
j
\end{array}\right) \text {, }
$$

we get

$$
\begin{aligned}
(N & +2 n+1) P_{2 n}(x)-(N+n) P_{2 n-1}(x) \cdot x \\
& =\sum_{j=0}^{n+1}(-1)^{j}\left(\begin{array}{c}
n+1 \\
j
\end{array}\right) \prod_{l=1}^{2 n-j+1}(N+l) \cdot x^{j} \\
& =P_{2 n+1} .
\end{aligned}
$$


Concerning $Q_{n}(x)$,

$$
\begin{aligned}
(N+ & 2 n) Q_{2 n-1}(x)+n Q_{2 n-2}(x) \cdot x \\
= & (N+2 n) \sum_{j=0}^{n-1} \sum_{k=0}^{j}(-1)^{j-k}(2 n-j-1)_{k}\left(\begin{array}{c}
n-k-1 \\
j-k
\end{array}\right) \prod_{l=k+1}^{2 n-j-1}(N+l) \cdot x^{j} \\
& +n \sum_{j=0}^{n-1} \sum_{k=0}^{j}(-1)^{j-k}(2 n-j-2)_{k}\left(\begin{array}{c}
n-k-2 \\
j-k
\end{array}\right) \prod_{l=k+1}^{2 n-j-2}(N+l) \cdot x^{j+1} \\
= & \prod_{l=1}^{2 n}(N+l)-n \sum_{k=0}^{n-1}(-1)^{n-k}(n)_{k}\left(\begin{array}{c}
n-k-2 \\
n-k-1
\end{array}\right) \prod_{l=k+1}^{n-1}(N+l) \cdot x^{n} \\
& +\sum_{j=1}^{n-1} \sum_{k=0}^{j}(-1)^{j-k}(2 n-j-1)_{k} \\
& \times\left(\begin{array}{l}
n-k-1 \\
j-k
\end{array}\right)(N+2 n) \prod_{l=k+1}^{2 n-j-1}(N+l) \cdot x^{j} \\
& -n \sum_{j=1}^{n-1} \sum_{k=0}^{j-1}(-1)^{j-k} n(2 n-j-1)_{k}\left(\begin{array}{c}
n-k-2 \\
j-k-1
\end{array}\right) \prod_{l=k+1}^{2 n-j-1}(N+l) \cdot x^{j}
\end{aligned}
$$

Since $N+2 n=(N+2 n-j)+j$,

$$
\begin{aligned}
& \prod_{l=k+1}^{2 n-j-1}(N+l)=\prod_{l=k+2}^{2 n-j}(N+l)-(2 n-j-k-1) \prod_{l=k+2}^{2 n-j-1}(N+l), \\
& (j-k)\left(\begin{array}{c}
n-k-1 \\
j-k
\end{array}\right)-n\left(\begin{array}{c}
n-k-2 \\
j-k-1
\end{array}\right)=-(k+1)\left(\begin{array}{c}
n-k-2 \\
j-k-1
\end{array}\right)
\end{aligned}
$$

and $(2 n-j-1)_{k}+k(2 n-j-1)_{k-1}=(2 n-j)_{k}$, we obtain

$$
\begin{aligned}
(N+ & 2 n) Q_{2 n-1}(x)+n Q_{2 n-2}(x) \cdot x \\
= & \prod_{l=1}^{2 n}(N+l)+n ! x^{n} \\
& +\sum_{j=1}^{n-1} \sum_{k=0}^{j}(-1)^{j-k}(2 n-j-1)_{k}\left(\begin{array}{c}
n-k-1 \\
j-k
\end{array}\right) \prod_{l=k+1}^{2 n-j}(N+l) \cdot x^{j} \\
& +\sum_{j=1}^{n-1} \sum_{k=0}^{j}(-1)^{j-k} j(2 n-j-1)_{k} \\
& \times\left(\begin{array}{l}
n-k-1 \\
j-k
\end{array}\right)^{(N+2 n) \prod_{l=k+1}^{2 n-j-1}(N+l) \cdot x^{j}} \\
& -\sum_{j=1}^{n-1} \sum_{k=0}^{j-1}(-1)^{j-k} n(2 n-j-1)_{k}
\end{aligned}
$$




$$
\begin{aligned}
& \times\left(\begin{array}{c}
n-k-2 \\
j-k-1
\end{array}\right)(N+2 n) \prod_{l=k+1}^{2 n-j-1}(N+l) \cdot x^{j} \\
= & \prod_{l=1}^{2 n}(N+l)+n ! x^{n} \\
& +\sum_{j=1}^{n-1} \sum_{k=0}^{j}(-1)^{j-k}(2 n-j)_{k}\left(\begin{array}{c}
n-k-1 \\
j-k
\end{array}\right) \prod_{l=k+1}^{2 n-j}(N+l) \cdot x^{j} \\
= & Q_{2 n} .
\end{aligned}
$$

Similarly,

$$
\begin{aligned}
(N+ & 2 n+1) Q_{2 n}(x)-(N+n) x Q_{2 n-1}(x) \\
= & (N+2 n+1) \sum_{j=0}^{n} \sum_{k=0}^{j}(-1)^{j-k}(2 n-j)_{k}\left(\begin{array}{c}
n-k-1 \\
j-k
\end{array}\right) \prod_{l=k+1}^{2 n-j}(N+l) \cdot x^{j} \\
& -(N+n) \sum_{j=0}^{n-1} \sum_{k=0}^{j}(-1)^{j-k}(2 n-j-1)_{k}\left(\begin{array}{c}
n-k-1 \\
j-k
\end{array}\right) \prod_{l=k+1}^{2 n-j-1}(N+l) \cdot x^{j+1} \\
= & (N+2 n+1) \prod_{l=k+1}^{2 n}(N+l) \\
& +\sum_{j=1}^{n} \sum_{k=0}^{j}(-1)^{j-k}(2 n-j)_{k}\left(\begin{array}{c}
n-k-1 \\
j-k
\end{array}\right) \prod_{l=k+1}^{2 n-j+1}(N+l) \cdot x^{j} \\
& \times \sum_{j=1}^{n} \sum_{k=0}^{j}(-1)^{j-k}(2 n-j)_{k} j\left(\begin{array}{c}
n-k-1 \\
j-k
\end{array}\right) \prod_{l=k+1}^{2 n-j}(N+l) \cdot x^{j} \\
& +\sum_{j=1}^{n} \sum_{k=0}^{j-1}(-1)^{j-k}(2 n-j)_{k}\left(\begin{array}{c}
n-k-1 \\
j-k-1
\end{array}\right) \prod_{l=k+1}^{2 n-j+1}(N+l) \cdot x^{j} \\
& -\sum_{j=1}^{n} \sum_{k=0}^{j-1}(-1)^{j-k}(2 n-j)_{k}(n-j+1)\left(\begin{array}{c}
n-k-1 \\
j-k-1
\end{array}\right) \prod_{l=k+1}^{2 n-j}(N+l) \cdot x^{j} .
\end{aligned}
$$

Since

$$
\left(\begin{array}{c}
n-k-1 \\
j-k
\end{array}\right)+\left(\begin{array}{c}
n-k-1 \\
j-k-1
\end{array}\right)=\left(\begin{array}{c}
n-k \\
j-k
\end{array}\right)
$$

and

$$
\begin{gathered}
j\left(\begin{array}{c}
n-k-1 \\
j-k
\end{array}\right)-(n-j+1)\left(\begin{array}{c}
n-k-1 \\
j-k-1
\end{array}\right) \\
=k\left(\begin{array}{c}
n-k \\
j-k
\end{array}\right)-(k+1)\left(\begin{array}{l}
n-k-1 \\
j-k-1
\end{array}\right),
\end{gathered}
$$

we get

$$
(N+2 n+1) Q_{2 n}(x)-(N+n) x Q_{2 n-1}(x)
$$




$$
\begin{aligned}
= & (N+2 n+1) \prod_{l=k+1}^{2 n}(N+l) \\
& +\sum_{j=1}^{n} \sum_{k=0}^{j}(-1)^{j-k}(2 n-j)_{k}\left(\begin{array}{c}
n-k \\
j-k
\end{array}\right) \prod_{l=k+1}^{2 n-j+1}(N+l) \cdot x^{j} \\
& +\sum_{j=1}^{n} \sum_{k=0}^{j}(-1)^{j-k}(2 n-j)_{k}\left(k\left(\begin{array}{c}
n-k \\
j-k
\end{array}\right)-(k+1)\left(\begin{array}{c}
n-k-1 \\
j-k-1
\end{array}\right)\right) \\
& \times \prod_{l=k+1}^{2 n-j}(N+l) \cdot x^{j} .
\end{aligned}
$$

Since

$$
\begin{aligned}
& (-1)^{j-k-1}(2 n-j)_{k+1}\left(\begin{array}{c}
n-k-1 \\
j-k-1
\end{array}\right) \prod_{l=k+2}^{2 n-j+1}(N+l) \\
& \quad+(-1)^{j-k}(2 n-j)_{k}\left(k\left(\begin{array}{c}
n-k \\
j-k
\end{array}\right)-(k+1)\left(\begin{array}{c}
n-k-1 \\
j-k-1
\end{array}\right)\right) \prod_{l=k+1}^{2 n-j}(N+l) \\
& \quad+(-1)^{j-k+1}(2 n-j)_{k}\left(\begin{array}{c}
n-k \\
j-k
\end{array}\right) \prod_{l=k+1}^{2 n-j}(N+l) \\
& =(-1)^{j-k-1}(2 n-j+1)_{k+1}\left(\begin{array}{c}
n-k-1 \\
j-k-1
\end{array}\right) \prod_{l=k+2}^{2 n-j+1}(N+l) \\
& \quad+(-1)^{j-k-1}(k+1)(2 n-j)_{k+1}\left(\begin{array}{c}
n-k-1 \\
j-k-1
\end{array}\right) \prod_{l=k+2}^{2 n-j}(N+l),
\end{aligned}
$$

we have

$$
\begin{aligned}
(N+ & 2 n+1) Q_{2 n}(x)-(N+n) x Q_{2 n-1}(x) \\
& +\sum_{j=1}^{n} \sum_{k=0}^{j}(-1)^{j-k}(2 n-j+1)_{k}\left(\begin{array}{c}
n-k \\
j-k
\end{array}\right) \prod_{l=k+1}^{2 n-j+1}(N+l) \cdot x^{j} \\
= & Q_{2 n+1}(x) .
\end{aligned}
$$

\subsection{Some more identities of hypergeometric Bernoulli numbers}

Since $P_{2 n-1}(x), P_{2 n}(x)$ and $Q_{2 n}(x)$ are polynomials with degree $n$ and $Q_{2 n-1}(x)$ is a polynomial with degree $n-1$, by the approximation property of the continued fraction, we have the following.

Lemma 8 Let $P_{n}(x) / Q_{n}(x)$ denote the $n$th convergent of the continued fraction expansion of (14). Then for $n \geq 0$,

$$
Q_{n}(x) \sum_{\kappa=0}^{\infty} B_{N, \kappa} \frac{x^{\kappa}}{\kappa !} \equiv P_{n}(x) \quad\left(\bmod x^{n+1}\right) .
$$


By this approximation property, the coefficients $x^{j}(0 \leq j \leq n)$ of

$$
Q_{n}(x) \sum_{\kappa=0}^{\infty} B_{N, \kappa} \frac{x^{\kappa}}{\kappa !}-P_{n}(x)
$$

are nullified. By Theorem 4,

$$
\begin{aligned}
Q_{2 n}(x) \sum_{\kappa=0}^{\infty} B_{N, \kappa} \frac{x^{\kappa}}{\kappa !} \\
\quad=\sum_{h=0}^{\infty} \sum_{j=0}^{\min \{h, n\}} \sum_{k=0}^{j}(-1)^{j-k}(2 n-j)_{k}\left(\begin{array}{c}
n-k-1 \\
j-k
\end{array}\right) \prod_{l=k+1}^{2 n-j}(N+l) \cdot \frac{B_{N, h-j}}{(h-j) !} x^{h}
\end{aligned}
$$

and

$$
P_{2 n}(x)=\sum_{h=0}^{n}(-1)^{h}\left(\begin{array}{l}
n \\
h
\end{array}\right) \prod_{l=1}^{2 n-h}(N+l) \cdot x^{h} .
$$

Therefore,

$$
\begin{aligned}
& \left.\sum_{j=0}^{\min \{h, n\}} \sum_{k=0}^{j}(-1)^{j-k}(2 n-j)\right)_{k}\left(\begin{array}{c}
n-k-1 \\
j-k
\end{array}\right) \prod_{l=k+1}^{2 n-j}(N+l) \cdot \frac{B_{N, h-j}}{(h-j) !} \\
& = \begin{cases}(-1)^{h}\left(\begin{array}{l}
n \\
h
\end{array}\right) \prod_{l=1}^{2 n-h}(N+l) & (0 \leq h \leq n) ; \\
0 & (h>n) .\end{cases}
\end{aligned}
$$

Similarly, since

$$
\begin{aligned}
Q_{2 n-1}(x) & \sum_{\kappa=0}^{\infty} B_{N, \kappa} \frac{x^{\kappa}}{\kappa !} \\
= & \sum_{h=0}^{\infty} \sum_{j=0}^{\min \{h, n-1\}} \sum_{k=0}^{j}(-1)^{j-k}(2 n-j-1)_{k}\left(\begin{array}{c}
n-k-1 \\
j-k
\end{array}\right) \\
& \times \prod_{l=k+1}^{2 n-j-1}(N+l) \cdot \frac{B_{N, h-j}}{(h-j) !} x^{h}
\end{aligned}
$$

and

$$
P_{2 n-1}(x)=\sum_{h=0}^{n}(-1)^{h}\left(\begin{array}{l}
n \\
h
\end{array}\right) \prod_{l=1}^{2 n-h-1}(N+l) \cdot x^{h}
$$

we have

$$
\sum_{j=0}^{\min \{h, n\}} \sum_{k=0}^{j}(-1)^{j-k}(2 n-j-1)_{k}\left(\begin{array}{c}
n-k-1 \\
j-k
\end{array}\right) \prod_{l=k+1}^{2 n-j-1}(N+l) \cdot \frac{B_{N, h-j}}{(h-j) !}
$$




$$
= \begin{cases}(-1)^{h}\left(\begin{array}{l}
n \\
h
\end{array}\right) \prod_{l=1}^{2 n-h-1}(N+l) & (0 \leq h \leq n) ; \\
0 & (h>n) .\end{cases}
$$

Theorem 5 We have

$$
\begin{gathered}
\sum_{j=0}^{\min \{h, n\}} \sum_{k=0}^{j}(-1)^{j-k}(2 n-j)_{k}\left(\begin{array}{c}
n-k-1 \\
j-k
\end{array}\right) \prod_{l=k+1}^{2 n-j}(N+l) \cdot \frac{B_{N, h-j}}{(h-j) !} \\
\quad= \begin{cases}(-1)^{h}\left(\begin{array}{l}
n \\
h
\end{array}\right) \prod_{l=1}^{2 n-h}(N+l) & (0 \leq h \leq n) ; \\
0 & (h>n),\end{cases}
\end{gathered}
$$

and

$$
\begin{gathered}
\sum_{j=0}^{\min \{h, n\}} \sum_{k=0}^{j}(-1)^{j-k}(2 n-j-1)_{k}\left(\begin{array}{c}
n-k-1 \\
j-k
\end{array}\right) \prod_{l=k+1}^{2 n-j-1}(N+l) \cdot \frac{B_{N, h-j}}{(h-j) !} \\
\quad= \begin{cases}(-1)^{h}\left(\begin{array}{l}
n \\
h
\end{array}\right) \prod_{l=1}^{2 n-h-1}(N+l) & (0 \leq h \leq n) ; \\
0 & (h>n) .\end{cases}
\end{gathered}
$$

In particular, when $N=1$, we have the relations for the classical Bernoulli numbers.

\section{Corollary 4 We have}

$$
\begin{aligned}
& \sum_{j=0}^{\min \{h, n\}} \sum_{k=0}^{j}(-1)^{j-k}(2 n-j)_{k}\left(\begin{array}{c}
n-k-1 \\
j-k
\end{array}\right) \frac{(2 n-j+1) !}{(k+1) !(2 n-h+1) !} \cdot \frac{B_{h-j}}{(h-j) !} \\
& \quad= \begin{cases}(-1)^{h}\left(\begin{array}{l}
n \\
h
\end{array}\right) & (0 \leq h \leq n) ; \\
0 & (h>n),\end{cases}
\end{aligned}
$$

and

$$
\begin{aligned}
& \sum_{j=0}^{\min \{h, n\}} \sum_{k=0}^{j}(-1)^{j-k}(2 n-j-1)_{k}\left(\begin{array}{c}
n-k-1 \\
j-k
\end{array}\right) \frac{(2 n-j) !}{(k+1) !(2 n-h) !} \cdot \frac{B_{h-j}}{(h-j) !} \\
& \quad= \begin{cases}(-1)^{h}\left(\begin{array}{l}
n \\
h
\end{array}\right) & (0 \leq h \leq n) ; \\
0 & (h>n) .\end{cases}
\end{aligned}
$$

Remark Since

$$
\begin{aligned}
& \sum_{k=0}^{j}(-1)^{j-k} \frac{(2 n-j)_{k}}{(k+1) !}\left(\begin{array}{c}
n-k-1 \\
j-k
\end{array}\right) \\
& \quad= \begin{cases}\frac{1}{j+1}\left(\begin{array}{c}
n \\
j
\end{array}\right) & \text { if } j \text { is even; } \\
\frac{1}{4 j}\left(\begin{array}{c}
j-2 \\
(j-1) / 2
\end{array}\right)^{-1}\left(\begin{array}{c}
n-(j+1) / 2 \\
(j-1) / 2
\end{array}\right)\left(\begin{array}{c}
n \\
(j-1) / 2
\end{array}\right) & \text { if } j \text { is odd } \geq 3 ; \\
\frac{1}{2} & \text { if } j=1\end{cases}
\end{aligned}
$$


we can write (19) as

$$
\begin{aligned}
& \sum_{j=0}^{\left\lfloor\frac{h}{2}\right\rfloor} \frac{(2 n-2 j+1) !}{2 j+1}\left(\begin{array}{c}
n \\
2 j
\end{array}\right) \frac{B_{h-2 j}}{(h-2 j) !}+\frac{(2 n) !}{2} \frac{B_{h-1}}{(h-1) !} \\
& \quad+\sum_{j=1}^{\left\lfloor\frac{h-1}{2}\right\rfloor} \frac{(2 n-2 j) !}{4(2 j+1)}\left(\begin{array}{c}
2 j-1 \\
j
\end{array}\right)\left(\begin{array}{c}
n-j-1 \\
j
\end{array}\right)\left(\begin{array}{c}
n \\
j
\end{array}\right) \frac{B_{h-2 j-1}}{(h-2 j-1) !} \\
& \quad= \begin{cases}(-1)^{h}\left(\begin{array}{l}
n \\
h
\end{array}\right)(2 n-h+1) ! & \text { if } 1 \leq h \leq n ; \\
0 & \text { if } n<h \leq 2 n+1 .\end{cases}
\end{aligned}
$$

Since

$$
\begin{aligned}
& \sum_{k=0}^{j}(-1)^{j-k} \frac{(2 n-j-1)_{k}}{(k+1) !}\left(\begin{array}{c}
n-k-1 \\
j-k
\end{array}\right) \\
& = \begin{cases}\frac{((j / 2) !)^{2}}{(j+1) !}\left(\begin{array}{c}
n \\
j / 2
\end{array}\right)\left(\begin{array}{c}
n-j / 2-1 \\
j / 2
\end{array}\right) & \text { if } j \text { is even; } \\
0 & \text { if } j \text { is odd, }\end{cases}
\end{aligned}
$$

we can write (20) as

$$
\begin{aligned}
& \sum_{j=0}^{\left\lfloor\frac{h}{2}\right\rfloor} \frac{(j !)^{2}(2 n-2 j) !}{(2 j+1) !}\left(\begin{array}{l}
n \\
j
\end{array}\right)\left(\begin{array}{c}
n-j-1 \\
j
\end{array}\right) \frac{B_{h-2 j}}{(h-2 j) !} \\
& \quad= \begin{cases}(-1)^{h}\left(\begin{array}{l}
n \\
h
\end{array}\right)(2 n-h) ! & \text { if } 1 \leq h \leq n ; \\
0 & \text { if } n<h \leq 2 n .\end{cases}
\end{aligned}
$$

Here the empty summation is recognized as 0 , as usual.

\section{Acknowledgements}

The authors would like to thank the referees for the helpful suggestions.

Funding

Not applicable.

\section{Availability of data and materials}

Not applicable.

\section{Competing interests}

The authors declare that they have no competing interests.

\section{Authors' contributions}

All authors contributed to the writing of this paper. All authors read and approved the final manuscript.

\section{Author details}

${ }^{1}$ Department of Mathematics, Shimane University, Matsue, Japan. ${ }^{2}$ Department of Mathematical Sciences, School of Science, Zhejiang Sci-Tech University, Hangzhou, China. ${ }^{3}$ National Institute of Technology, Rourkela, India.

\section{Publisher's Note}

Springer Nature remains neutral with regard to jurisdictional claims in published maps and institutional affiliations.

Received: 6 January 2019 Accepted: 11 April 2019 Published online: 24 April 2019 


\section{References}

1. Arakawa, T., Ibukiyama, T., Kaneko, M.: Bernoulli Numbers and Zeta Functions. Springer Monographs in Mathematics. Springer, Tokyo (2014) With an appendix by Don Zagier

2. Brioschi, F.: Sulle funzioni Bernoulliane ed Euleriane. Ann. Mat. Pura Appl. (1858-1865) 1, 260-263 (1858)

3. Carlitz, L.: On certain functions connected with polynomials in a Galois field. Duke Math. J. 1, 137-168 (1935)

4. Carlitz, L: q-Bernoulli numbers and polynomials. Duke Math. J. 15, 987-1000 (1948)

5. Carlitz, L.: Some theorems on Bernoulli numbers of higher order. Pac. J. Math. 2, 127-139 (1952)

6. Carlitz, L.: Degenerate Stirling, Bernoulli and Eulerian numbers. Util. Math. 15, 51-88 (1979)

7. Glaisher, J.W.L.: Expressions for Laplace's coefficients, Bernoullian and Eulerian numbers etc. as determinants. Messenger 6, 49-63 (1875)

8. Gottfert, R., Niederreiter, H.: Hasse-Teichmüller derivatives and products of linear recurring sequences. In: Finite Fields: Theory, Applications, and Algorithms, Las Vegas, 1993. Contemporary Mathematics, vol. 168, pp. 117-125. American Mathematical Society, Providence (1994)

9. Graham, R.L., Knuth, D.E., Patashnik, O.: Concrete Mathematics, a Foundation for Computer Science, 2nd edn. Addison-Wesley, Reading (1994)

10. Hamahata, Y., Masubuchi, H.: Special multi-poly-Bernoulli numbers. J. Integer Seq. 10, Article ID 07.4.1 (2007)

11. Hamahata, Y., Masubuchi, H.: Recurrence formulae for multi-poly-Bernoulli numbers. Integers 7, A46 (2007)

12. Hasse, H.: Theorie der höheren Differentiale in einem algebraischen Funktionenkörper mit Vollkommenem Konstantenkörper bei beliebiger Charakteristik. J. Reine Angew. Math. 175, 50-54 (1936)

13. Hassen, A., Nguyen, H.D.: Hypergeometric Bernoulli polynomials and Appell sequences. Int. J. Number Theory 4 767-774 (2008)

14. Hassen, A., Nguyen, H.D.: Hypergeometric zeta functions. Int. J. Number Theory 6, 99-126 (2010)

15. Howard, F.T: A sequence of numbers related to the exponential function. Duke Math. J. 34, 599-615 (1967)

16. Howard, F.T: Some sequences of rational numbers related to the exponential function. Duke Math. J. 34, 701-716 (1967)

17. Hu, S., Kim, M.-S.: On hypergeometric Bernoulli numbers and polynomials. Acta Math. Hung. 154, 134-146 (2018)

18. Kamano, K.: Sums of products of hypergeometric Bernoulli numbers. J. Number Theory 130, 2259-2271 (2010)

19. Kaneko, M.: A recurrence formula for the Bernoulli numbers. Proc. Jpn. Acad., Ser. A, Math. Sci. 71, 192-193 (1995)

20. Kaneko, M.: Poly-Bernoulli numbers. J. Théor. Nr. Bordx. 9, 221-228 (1997)

21. Komatsu, T., Ramirez, J.L.: Some determinants involving incomplete Fubini numbers. An. Ştiinţ. Univ. 'Ovidius' Constanța, Ser. Mat. 26(3), 143-170 (2018). https://doi.org/10.2478/auom-2018-0038

22. Komatsu, T., Yuan, P.: Hypergeometric Cauchy numbers and polynomials. Acta Math. Hung. 153, 382-400 (2017)

23. Luo, Q.-M.: An explicit relationship between the generalized Apostol-Bernoulli and Apostol-Euler polynomials associated with $\lambda$-Stirling numbers of the second kind. Houst. J. Math. 36, 1159-1171 (2010)

24. Muir, T.: The Theory of Determinants in the Historical Order of Development, Four Volumes. Dover Publications, New York (1960)

25. Nguyen, H.D.: Sums of products of hypergeometric Bernoulli polynomials. In: MAA-NJ Section—Spring Meeting, Middle Country College, NJ (2010)

26. Nguyen, H.D., Cheong, L.G.: New convolution identities for hypergeometric Bernoulli polynomials. J. Number Theory 137, 201-221 (2014)

27. Teichmüller, O.: Differentialrechung bei Charakteristik p. J. Reine Angew. Math. 175, 89-99 (1936)

28. Trudi, N.: Intorno ad alcune formole di sviluppo. In: Rendic. dell' Accad. Napoli, pp. 135-143 (1862)

29. Wall, H.S.: Analytic Theory of Continued Fractions. Chelsea Publ., New York (1948)

30. Washington, L.: Introduction to Cyclotomic Fields, 2nd edn. Graduate Texts in Mathematics, vol. 83. Springer, Berlin (1997)

\section{Submit your manuscript to a SpringerOpen ${ }^{\circ}$ journal and benefit from:}

- Convenient online submission

- Rigorous peer review

- Open access: articles freely available online

- High visibility within the field

- Retaining the copyright to your article

Submit your next manuscript at $\gg$ springeropen.com 